\title{
A TRULY-MESHLESS GALERKIN METHOD, THROUGH THE MLPG "MIXED" APPROACH
}

\section{Zhidong Han}

Department of Mechanical and Aerospace Engineering, University of California, Irvine, CA, U.S.A.

Satya N. Atluri

Department of Mechanical and Aerospace Engineering, University of California, Irvine, CA, U.S.A., satluri@uci.edu

Follow this and additional works at: https://jmstt.ntou.edu.tw/journal

Part of the Engineering Commons

\section{Recommended Citation}

Han, Zhidong and Atluri, Satya N. (2011) "A TRULY-MESHLESS GALERKIN METHOD, THROUGH THE MLPG "MIXED" APPROACH," Journal of Marine Science and Technology. Vol. 19: Iss. 4, Article 14.

DOI: 10.51400/2709-6998.2187

Available at: https://jmstt.ntou.edu.tw/journal/vol19/iss4/14

This Research Article is brought to you for free and open access by Journal of Marine Science and Technology. It has been accepted for inclusion in Journal of Marine Science and Technology by an authorized editor of Journal of Marine Science and Technology. 


\title{
A TRULY-MESHLESS GALERKIN METHOD, THROUGH THE MLPG “MIXED” APPROACH
}

\author{
Zhidong Han* and Satya N. Atluri*
}

Key words: Meshless Local Petrov-Galerkin approach (MLPG), Galerkin Methods, Mixed Methods.

\begin{abstract}
A truly meshless Galerkin method is formulated in the present study, as a special case of the general Meshless Local Petrov-Galerkin (MLPG) "Mixed" approach. The Galerkin method is implemented as a truly meshless method, for solving elasto-static problems. In the present Galerkin method, the test function is chosen to be the same as the trial function, as a special case of the MLPG approach. However, the MLPG local weak form is written over a local sub-domain which is completely independent from the trial or test functions. Even though in the present Galerkin approach, the trial and test functions are the same, the present MLPG approach (wherein the support sizes of the nodal trial and test function domains, as well as the size of the local subdomain over which the local weak-form is considered, can be arbitrary) may lead to either symmetric or unsymmetric "stiffness" matrices. These matrices are sparse and are well-conditioned. The present MLPG Galerkin Mixed Method does not require any background meshes (or cells) for performing the numerical integration of the local weak-forms, and makes the present method to be truly meshless. In addition, the mixed approach is also used to interpolate the nodal values of strains independently from the nodal values of displacements. The present mixed approach eliminates the expensive process of directly differentiating the interpolations for displacements in the entire domain, to find the derivatives, such as strains and stresses. The present MLPG Galerkin Mixed Method is not plagued by the so-called LBB conditions, which are common in the Galerkin Mixed Finite Element Method. Numerical examples are included to demonstrate the advantages of the present method: i) the truly meshless implementation; ii) the simplicity of the mixed approach wherein lower-order polynomial basis and smaller support sizes can be used; and iii) higher accuracies and computational efficiencies, and iv) no LBB conditions.
\end{abstract}

Paper submitted 11/04/10; revised 12/15/10; accepted 01/14/11. Author for correspondence: Satya N. Atluri (e-mail: satluri@uci.edu).

*Department of Mechanical and Aerospace Engineering, University of California, Irvine, CA, U.S.A.

\section{INTRODUCTION}

It is well known that the meshless methods have advantages over the traditional mesh-based methods, in overcoming the drawbacks of mesh-based methods, such as the labor-intensive process of mesh-generation, locking, poor derivative solutions, etc. The meshless methods may also eliminate the mesh distortion problems once the solid/structure undergoes large deformations, in which case, adaptive refinement and adaptive remeshing are required. Several meshless methods have been developed based on the global weak forms, in which simply the element-based trial and test functions are replaced by certain meshless interpolations. They still require certain meshes or background cells for performing the global integrals of the global weak forms. These requirements limit such globalweak-form based meshless methods from being truly meshless for eliminating the mesh distortion problems coupled with the large deformations.

In contrast, the meshless local Petrov-Galerkin (MLPG) approach pioneered by Atluri and his colleagues $[1,7,8]$ is based on several different local weak forms of PDEs over overlapping local sub-domains. It uses meshless interpolations for the trial and test functions, and performs the integrations of the local-weak-forms within the local sub-domains. Thus the MLPG approach becomes a general frame work for developing truly meshless methods for solving various problems, especially for those with severe distortion, discontinuities, and moving boundaries. Because of the lack of nodal connectivity, and its truly meshless nature, the MLPG method is a natural candidate for parallelization and high-performance computing based algorithms. Various methods have been developed based on the MLPG approach, including the primal MLPG method [8], the finite volume method [7], Local BIE [4], the Mixed finite volume method [2], the mixed collocation method [5], the mixed finite difference method [6], the primal MLPG method for 3-D problems [9, 10], the MLPG BIE [3, 11-13], the MLPG for fluid mechanics [16], and many others as summarized in Atluri [1].

After the pioneering work on the MLPG approach by Alturi and Han [8], the mixed approach has been pioneered by Atluri et al. [2], in which the derivatives of the variables are independently interpolated, rather than obtaining the derivatives by directly differentiating the primal variables themselves. As an example, in solid mechanics problems, the independent 
meshless approximations are used for both the strains, as well as the displacements. The strain-displacement compatibility is enforced only at the nodal points in the local approximation, by using the collocation method; thus expressing the independent nodal strains in terms of nodal displacements. Theoretically, the MLPG mixed approach requires that the trial functions possess only $\mathrm{C} 0$ continuity, if the symmetrical weak forms are used for the elasto-elastic problems. In contrast, $\mathrm{C} 1$ continuities are required for the trial functions if the primal approach is used, and the strains everywhere are derived by differentiating the primary variables (displacements, if using the symmetric weak form in solid mechanics). In the numerical implementation of the mixed methods, the strains are interpolated directly via the meshless approximations, without the calculation of the derivatives of the shape functions. The mixed approach is thus computationally more efficient, because the calculation of the derivatives of the interpolation functions in the meshless approximations is computationally costly. In addition, in the primal approach the second-order polynomial bases are required for the better approximation of displacement, in order to avoid shear-locking if the MLS is used [9]. Also in the MLPG primal methods, a larger support size should be chosen, in order to make the MLS approximation non-singular, which leads to over-smoothed results. However, the mixed approach requires only a firstorder polynomial basis in the MLS approximations of both strains as well as displacements. A smaller support size can be used in the mixed approach, and the number of nodes is reduced dramatically, especially for 3D cases. Furthermore, in the MLPG mixed approach in solid mechanics, as presented for the Galerkin method in the present paper, and as presented for the Finite Volume Method in [2], there are no LBB stability conditions to be satisfied, as in the case of mixed Finite Element Methods based on Global variational principles of the saddle-point type. The MLPG meshless mixed approach has been widely applied in various problems for solid mechanics $[1,2,5,6]$, thick plates $\&$ shells $[14,15]$, breaking waves $[18$, 24], ill-posed linear problems [17], elastic transient problems [19], anisotropic solids [20-22].

In the present study, the truly meshless Galerkin method is developed as a special case of the MLPG approach. The trial and test functions are chosen to be the same, which leads to a symmetric, semi-definite system of equations, if the support sizes of the trial and test functions are the same, and equal to the size of the local subdomain wherein the local weak-form is evaluated. However, the local sub-domain is completely decoupled from the nodal support domain, which is the major difference between the present method and the other Galerkin methods. The advantage here is the local sub-domain can be chosen to be of an arbitrary shape and size, to simplify the implementation and/or speed up the performance. All integrations are also performed within the local subdomain without any global mesh or background cells. It becomes very flexible and truly meshless. However, it introduces an extra integral term along the boundary of the subdomain, which

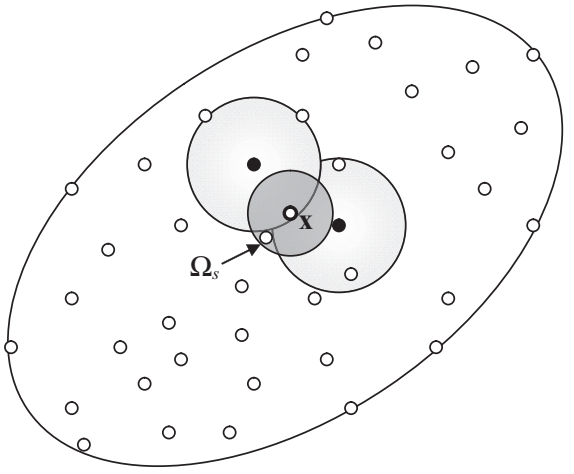

Fig. 1. A local sub-domain around point $x$.

normally vanishes if the local subdomain is chosen to be the same as the support domain. In the present study, the mixed approach is also used to reduce the size of the support domain. Due to the possible symmetric system of equations, the present Galerkin method can be connected with any element-based methods, especially when elements become distorted severely.

The main body of the paper begins with a brief introduction of the meshless MLS approximations in Section 2. The truly meshless Galerkin method is formulated through the MLPG approach in Section 3. The mixed approach and numerical discretization are presented in Section 4, and that symmetric and un-symmetric system matrices may arise out of the present truly meshless Gaerkin approach is illustrated in Section 5. Some numerical implementation techniques with the pseudo codes are given in Section 6. Numerical examples are given in Section 7, and the conclusions and discussions are given in Section 8 .

\section{MESHLESS APPROXIMATIONS}

For illustration purposes, one type of a general meshless interpolation, the moving least squares (MLS), is used in the present study, while a variety of alternate meshless interpolations such as Local/Global RBF, PU, RKPM, etc. may also be chosen. The MLS method of interpolation is generally considered to be one of the simplest schemes to interpolate random data with a reasonable accuracy, because of its completeness, robustness and continuity. More details can be found in Alturi [1].

With the MLS, the distribution of a function $u$ in $\Omega_{s}$ can be approximated, over a number of scattered local points $\left\{\mathbf{x}_{i}\right\}$, $(i=1,2, \ldots, n)$, as,

$$
u(\mathbf{x})=\mathbf{p}^{T}(\mathbf{x}) \mathbf{a}(\mathbf{x}) \quad \forall \mathbf{x} \in \Omega_{s}
$$

where $\mathbf{p}^{T}(\mathbf{x})=\left[p_{1}(\mathbf{x}), p_{2}(\mathbf{x}), \ldots, p_{m}(\mathbf{x})\right]$ is a monomial basis of order $m$; and $\mathbf{a}(\mathbf{x})$ is a vector containing coefficients, which are functions of the global Cartesian coordinates $\left[x_{1}, x_{2}, x_{3}\right]$, depending on the monomial basis. They are determined by minimizing a weighted discrete $L_{2}$ norm, defined, as: 


$$
\begin{aligned}
& J(\mathbf{x})=\sum_{i=1}^{m} w_{i}(\mathbf{x})\left[\mathbf{p}^{T}\left(\mathbf{x}_{i}\right) \mathbf{a}(\mathbf{x})-\hat{u}_{i}\right]^{2} \\
& \equiv[\mathbf{P} \cdot \mathbf{a}(\mathbf{x})-\hat{\mathbf{u}}]^{T} \mathbf{W}[\mathbf{P} \cdot \mathbf{a}(\mathbf{x})-\hat{\mathbf{u}}]
\end{aligned}
$$

where $w_{i}(\mathbf{x})$ are the weight functions and $\hat{u}_{i}$ are the fictitious nodal values.

One may obtain the shape function as,

$$
u(\mathbf{x})=\mathbf{p}^{T}(\mathbf{x}) \mathbf{A}^{-1}(\mathbf{x}) \mathbf{B}(\mathbf{x}) \hat{\mathbf{u}} \equiv \boldsymbol{\Phi}^{T}(\mathbf{x}) \hat{\mathbf{u}} \quad \forall \mathbf{x} \in \partial \Omega_{x}
$$

where matrices $\mathbf{A}(\mathbf{x})$ and $\mathbf{B}(\mathbf{x})$ are defined by

$$
\mathbf{A}(\mathbf{x})=\mathbf{P}^{T} \mathbf{W P} \quad \mathbf{B}(\mathbf{x})=\mathbf{P}^{T} \mathbf{W} \quad \forall \mathbf{x} \in \partial \Omega_{x}
$$

\section{THE TRULY MESHLESS GALERKIN METHOD THROUGH THE MLPG APPROACH}

Consider a linear elastic body in a $3 \mathrm{D}$ domain $\Omega$, with a boundary $\partial \Omega$. The solid is assumed to undergo infinitesimal deformations. The equations of the balances of linear and angular momentum can be written as:

$$
\sigma_{i j, j}+f_{i}=0 ; \sigma_{i j}=\sigma_{j i} ;()_{, i} \equiv \frac{\partial}{\partial \xi_{i}}
$$

where $\sigma_{i j}$ is the stress tensor, which corresponds to the displacement field $u_{i} ; f_{i}$ is the body force. The corresponding boundary conditions are given as follows,

$$
\begin{gathered}
u_{i}=\bar{u}_{i} \text { on } \Gamma_{u} \\
t_{i} \equiv \sigma_{i j} n_{j}=\bar{t}_{i} \text { on } \Gamma_{t}
\end{gathered}
$$

where $\bar{u}_{i}$ and $\bar{t}_{i}$ are the prescribed displacements and tractions, respectively, on the displacement boundary $\Gamma_{u}$ and on the traction boundary $\Gamma_{t}$, and $n_{i}$ is the unit outward normal to the boundary $\Gamma$.

In the MLPG approaches, one may write a weak form over a local sub-domain $\Omega_{s}$, which may have an arbitrary shape, and contain the point $\mathbf{x}$ in question. A generalized local weak form of the differential Eq. (5) over a local sub-domain $\Omega_{s}$, can be written as:

$$
\int_{\Omega_{s}}\left(\sigma_{i j}\left\{u_{k}\right\}_{, j}+f_{i}\right) v_{i} d \Omega=0
$$

where $u_{i}$ and $v_{i}$ are the trial and test functions, respectively, and $\sigma_{i j}\left\{u_{k}\right\}$ implies that $\sigma_{i j}$ are derived from the trial functions $u_{k}$.

By applying the divergence theorem, Eq. (7) may be rewritten in a symmetric weak form as:

$$
\int_{\partial \Omega_{s}} \sigma_{i j} n_{j} v_{i} d \Gamma-\int_{\Omega_{s}}\left(\sigma_{i j} v_{i, j}-f_{i} v_{i}\right) d \Omega=0
$$

Imposing the traction boundary conditions in Eq. (6), one obtains

$$
\begin{gathered}
\int_{L_{s}} t_{i} v_{i} d \Gamma+\int_{\Gamma_{s u}} t_{i} v_{i} d \Gamma+\int_{\Gamma_{s t}} \bar{t}_{i} v_{i} d \Gamma \\
-\int_{\Omega_{s}}\left(\sigma_{i j} v_{i, j}-f_{i} v_{i}\right) d \Omega=0
\end{gathered}
$$

where $\Gamma_{s u}$ is a part of the boundary $\partial \Omega_{s}$ of $\Omega_{s}$, over which the essential boundary conditions are specified. In general, $\partial \Omega_{s}=$ $\Gamma_{s} \cup L_{s}$ with $\Gamma_{\mathrm{s}}$ being a part of the local boundary located on the global boundary, and $L_{s}$ is the other part of the local boundary which is inside the solution domain. $\Gamma_{s u}=\Gamma_{s} \cap \Gamma_{u}$ is the intersection between the local boundary $\partial \Omega_{s}$ and the global displacement boundary $\Gamma_{u} ; \Gamma_{s t}=\Gamma_{s} \cap \Gamma_{t}$ is a part of the boundary over which the natural boundary conditions are specified.

Therefore, a local symmetric weak form (LSWF) in linear elasticity can be written as:

$$
\begin{gathered}
\int_{\Omega_{s}} \sigma_{i j} v_{i, j} d \Omega-\int_{L_{s}} t_{i} v_{i} d \Gamma-\int_{\Gamma_{s u}} t_{i} v_{i} d \Gamma \\
=\int_{\Gamma_{s t}} \overline{t_{i}} v_{i} d \Gamma+\int_{\Omega_{s}} f_{i} v_{i} d \Omega
\end{gathered}
$$

By choosing the test function $v_{i}$ to be identical to the trial function $u_{i}$, the local symmetric weak form in Eq. (10), and may be written as,

$$
\begin{gathered}
\int_{\Omega_{s}} \sigma_{i j} u_{i, j} d \Omega-\int_{L_{s}} t_{i} u_{i} d \Gamma-\int_{\Gamma_{s u}} t_{i} u_{i} d \Gamma \\
=\int_{\Gamma_{s t}} \bar{t}_{i} u_{i} d \Gamma+\int_{\Omega_{s}} f_{i} u_{i} d \Omega
\end{gathered}
$$

Eq. (11) has the physical meaning that it represents the variational statement only over the local subdomains, instead of over the global domain as it is for the finite element methods. It needs to be pointed out that: i) the local domains are completely independent from the trial and test functions; ii) they can be over-lapping or non-over-lapping; iii) they do not need any background mesh or cells for the numerical integration.

In the primal approach, $\sigma_{i j}$ in Eq. (11) will be expressed directly in terms of $u_{i}$, by differentiating $u_{i}$ analytically to find the strain $\varepsilon_{i j}=\frac{1}{2}\left(u_{i, j}+u_{j, i}\right)$ and by relating stress $\sigma_{i j}$ to $\varepsilon_{i j}$ through the material constitutive law. However, in the Mixed Galerkin approach as developed in the present paper, both $\varepsilon_{i j}$ as well as $u_{i}$ are directly interpolated locally over a small set of nodes, using independent equal order meshless approximations. This, however, does not involve any LBB conditions of stability, as in the case of the Global Finite Element Methods 


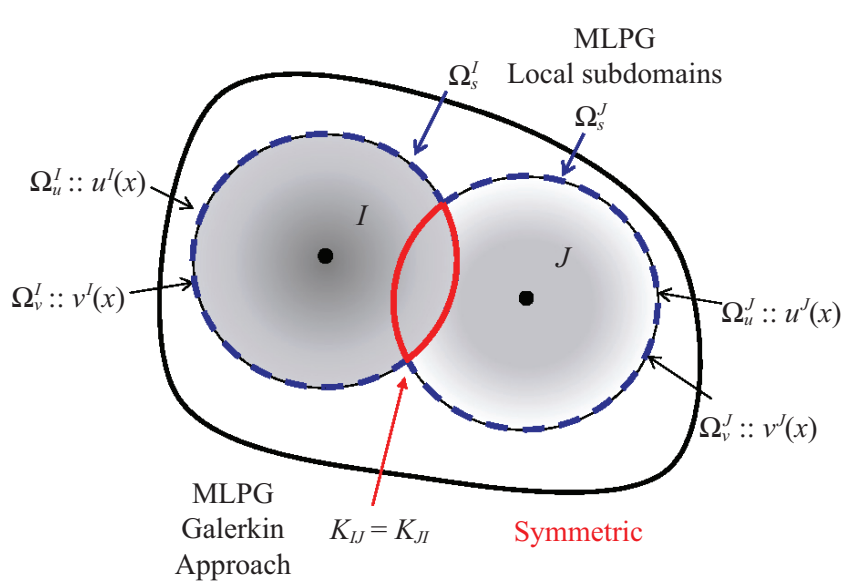

Fig. 2. Symmetric Galerkin Method.

based on Reissner's variational principle involving $\sigma_{i j}$ (or $\varepsilon_{i j}$ ) and $u_{i}$ as independent variables.

\section{THE POSSIBILITY OF EITHER SYMMETRIC OR UN-SYMMETRIC SYSTEM MATRICES IN THE TRULY MESHLESS GALERKIN METHOD}

In most truly meshless Galerkin methods, which is developed here as a special case of the most general MLPG method, the trial and test functions are chosen to be the same, and the size of the local sub-domain is also chosen to be the same as the size of the support domain. As shown in Fig. 2, the sizes of the support domain $\Omega_{u}^{I}$ of the trial function $u^{I}(x)$, the test domain $\Omega_{v}^{I}$ of the test function $v^{I}(x)$, and the local sub-domain $\Omega_{s}^{I}$ of node $I$ are exactly same. It is also same for node $J$. The local integral between nodes $I$ and $J$ of the Galerkin approach can be written as:

$$
\begin{aligned}
& K_{I J}=\int_{\Omega_{s}^{I} \cap \Omega_{u}^{J}} f\left[v^{I}(x), u^{J}(x)\right] d \Omega \\
& K_{J I}=\int_{\Omega_{s}^{J} \cap \Omega_{u}^{I}} f\left[u^{I}(x), v^{J}(x)\right] d \Omega
\end{aligned}
$$

with the consideration of

$$
\begin{array}{ll}
\Omega_{s}^{I}=\Omega_{u}^{I} & u^{I}(x)=v^{I}(x) \\
\Omega_{s}^{J}=\Omega_{u}^{J} & u^{J}(x)=v^{J}(x)
\end{array}
$$

Thus

$$
K_{I J}=K_{J I}
$$

However, in the present truly meshless Galerkin method, the local sub-domain $\Omega_{s}$ can be arbitrary, and may be smaller

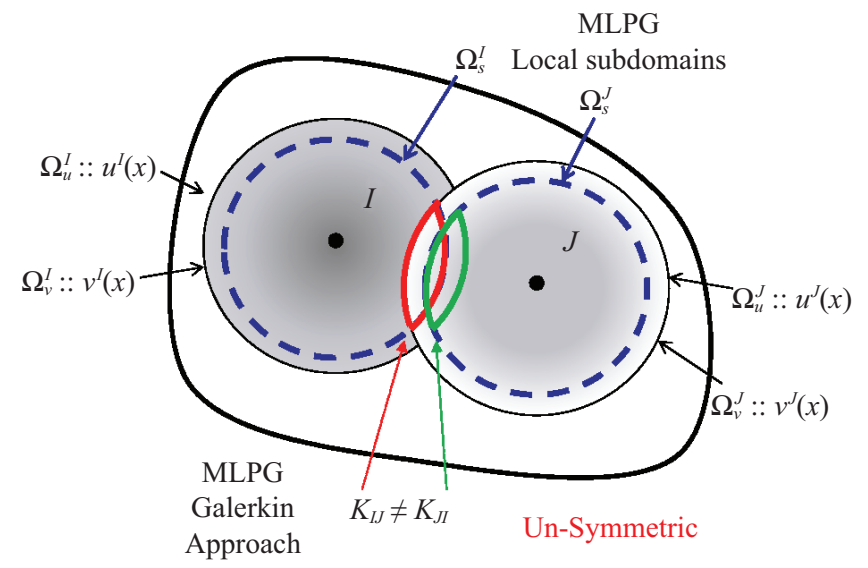

Fig. 3. Un-Symmetric Galerkin Method.

in size than the support domain $\Omega_{u}$ and the trial domain $\Omega_{v}$, as shown in Fig. 3. Therefore, the intersections between the local domains are different for $K_{I J}$ and $K_{J I}$, as

$$
K_{I J}=\int_{\Omega_{s}^{I} \cap \Omega_{u}^{J}} f\left[v^{I}(x), u^{J}(x)\right] d \Omega \text {; the integration is performed }
$$
over $\Omega_{s}^{I} \cap \Omega_{u}^{J}$ (the red zone in Fig. 3)

$K_{J I}=\int_{\Omega_{s}^{J} \cap \Omega_{u}^{I}} f\left[u^{I}(x), v^{J}(x)\right] d \Omega$; the integration is performed over $\Omega_{s}^{J} \cap \Omega_{u}^{I}$ (the green zone in Fig. 3)

It is very clear that they are not equal, which leads to un-symmetric system matrices. In the present study, the local sub-domain is chosen to be smaller than the support domain, and the numerical examples are calculated based on the unsymmetric, sparse, and well-conditioned, system matrices.

\section{THE MIXED APPROACH AND NUMERICAL DISCRETIZATION}

Using the constitutive relations of an isotropic linear elastic homogeneous solid for example, the stresses and tractions in Eq. (11) can be written in term of the strains:

$$
\sigma_{i j}=E_{i j k l} \varepsilon_{k l} \text { and } t_{i}=\sigma_{i j} n_{j}=E_{i j k l} \varepsilon_{k l} n_{j}
$$

where,

$$
E_{i j k l}=\lambda \delta_{i j} \delta_{k l}+\mu\left(\delta_{i k} \delta_{j l}+\delta_{i l} \delta_{j k}\right)
$$

with $\lambda$ and $\mu$ being the Lame's constants.

Consider a local sub-domain $\Omega_{s}$, centered on each nodal point $\mathbf{x}^{(I)}$; then the approximation of traction vectors on the boundary of $\Omega_{s}$ can be expressed by considering the nodal strains as independent variables. With the use of the shape function in Section 2, the strains are independently interpolated, as, 


$$
\varepsilon_{k l}(\mathbf{x})=\sum_{K=1}^{N} \Phi^{(K)}(\mathbf{x}) \varepsilon_{k l}^{(K)}
$$

In the present truly meshless Mixed Galerkin MLPG method, when the displacements and strains are independently interpolated as in Eqs. (3) and (16), respectively, using Eq. (14), we may write the discretized form of Eq. (11), as

$$
\begin{aligned}
& \sum_{L=1}^{N} \varepsilon_{i j}^{(L)} \sum_{K=1}^{N}\left[\int_{\Omega_{s}} \Phi^{(L)}(\mathbf{x}) \Phi^{(K)}(\mathbf{x}) E_{i j k l} d \Omega\right] \varepsilon_{k l}^{(K)} \\
& \quad-\sum_{L=1}^{N} u_{i}^{(L)} \sum_{K=1}^{N}\left[\int_{L_{s}} \Phi^{(L)}(\mathbf{x}) \Phi^{(K)}(\mathbf{x}) E_{i j k l} n_{j} d \Gamma\right] \varepsilon_{k l}^{(K)} \\
& \quad-\sum_{L=1}^{N} u_{i}^{(L)} \sum_{K=1}^{N}\left[\int_{\Gamma_{s u}} \Phi^{(L)}(\mathbf{x}) \Phi^{(K)}(\mathbf{x}) E_{i j k l} n_{j} d \Gamma\right] \varepsilon_{k l}^{(K)} \\
& =\sum_{L=1}^{N} u_{i}^{(L)} \int_{\Gamma_{s t}} \Phi^{(L)}(\mathbf{x}) \bar{t}_{i} d \Gamma \\
& \quad+\sum_{L=1}^{N} u_{i}^{(L)} \int_{\Omega_{s}} \Phi^{(L)}(\mathbf{x}) f_{i} d \Omega
\end{aligned}
$$

It clearly shows that no derivatives of the shape functions are involved in the local integrals $[2,5,6]$. The mixed approach speeds up the performance by avoiding the time-costly algorithms in calculating derivatives of the meshless interpolated trial functions. It has been verified that the efficiency of the mixed approach is improved over the traditional MLPG [primal] displacement methods. The mixed approach also reduces the requirement of the completeness and continuity of the shape functions by one-order, because the strains, which are the secondary field variables, are approximated independently of the displacements. Thus, lower-order polynomial terms are required in the meshless approximations, and a smaller nodal influence size can be chosen, to speed up the calculation of the shape functions. On the other hand, the number of equations in Eq. (17) is less than the number of the independent strain variables, because the nodal strain variables are more than the displacement ones [in 3D, there are six nodal-strain variables, but only 3 displacement nodal-variables]. One may follow the procedures in the original steps proposed by Atluri et al. [2] to reduce the number of the variables by transforming the strain variables back to the displacement variables via the collocation methods, without any changes to Eq. (17). First, the interpolation of displacements can also be accomplished by using the same shape function, from the nodal displacement variables, and written as,

$$
u_{i}(\mathbf{x})=\sum_{J=1}^{N} \Phi^{(J)}(\mathbf{x}) u_{i}^{(J)}
$$

For linear elasto-statics, the strain-displacement relations are:

$$
\varepsilon_{k l}=\frac{1}{2}\left(u_{k, l}+u_{l, k}\right)
$$

The standard collocation method may be applied to enforce Eq. (19) only at each nodal point $\mathbf{x}^{(I)}$, instead of the entire solution domain. Thus, the nodal strain variables are expressed in terms of the nodal displacement variables, as

$$
\varepsilon_{k l}\left(\mathbf{x}^{(I)}\right)=\frac{1}{2}\left[u_{k, l}\left(\mathbf{x}^{(I)}\right)+u_{l, k}\left(\mathbf{x}^{(I)}\right)\right]
$$

With the displacement approximation in Eq. (18), the two sets of nodal variables can be transformed through a linear algebraic matrix:

$$
\varepsilon_{k l}^{(I)}=H_{k l m}^{(I)(J)} u_{m}^{(J)}
$$

where the transformation matrix $H$ is banded.

The number of system equations is then reduced to the same number as the nodal-displacement variables, after the transformation. In addition, such a transformation is performed locally, and the system matrix retains its bandedness. For numerical implementation, it is not necessary to calculate and store the matrix $H$ explicitly. The integrals in Eq. (17) are only related to a few nodal points which are near to the point of interestion, $\mathbf{x}^{(I)}$, which means only a very small portion of the transformation matrix $H$ is used. It is possible to calculate this portion from Eq. (21) dynamically, which is less computationally costly because only a few local nodal points are involved.

In the present study, the collocation method is used to impose the essential displacement boundary conditions. For a nodal point $\mathbf{x}^{(I)}$, if its $i$ th displacement DOF belongs to the displacement-prescribed boundary-segment, i.e., $u_{i}^{(I)} \in \Gamma_{s u}$, the corresponding system equation can be replaced by the one generated from the collocation for this particular DOF, as

$$
\alpha u_{i}\left(\mathbf{x}^{(I)}\right)=\alpha \bar{u}_{i}\left(\mathbf{x}^{(I)}\right)
$$

or

$$
\alpha \sum_{L=1}^{N} u_{i}^{(L)} \sum_{K=1}^{N}\left[\int_{\Omega_{s}} \Phi^{(L)}\left(\mathbf{x}^{(I)}\right) \Phi^{(K)}\left(\mathbf{x}^{(I)}\right)\right] u_{i}^{(K)}=\alpha \bar{u}_{i}\left(\mathbf{x}^{(I)}\right)
$$

This standard collocation still keeps the system equations symmetric, sparse and banded.

It should be pointed out that the present method is formulated based on the nodal points fully within the local sub-domains, as shown in Eqs. (17), (21) and (22).

\section{NUMERICAL IMPLEMENTATION}

In the present study, the numerical implementation also 


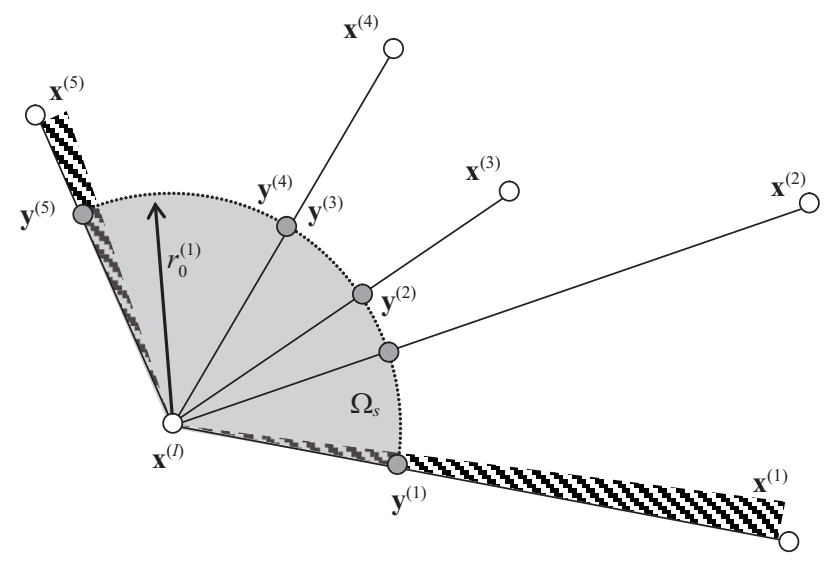

Fig. 4. A local sub-domain around point $x$.

follows the same steps as in the truly meshless implementation of the MLPG mixed finite volume method, reported in [2]. The only difference is that there is a domain integral in Eq. (11). First, we use a simple subdivision algorithm to simplify the numerical quadrature and improve the accuracy.

Consider a local circular sub-domain centered at node $I, \mathbf{x}^{(I)}$, with a radius denoted by $r_{0}^{(I)}$. By drawing a line from node $I$ to its neighbor node $J,\left\{\mathbf{x}^{(J)}\right\},(\mathrm{J}=1,2, \ldots, m)$, a point can be obtained at the intersection between the line and the local circle, denoted by $\left\{\mathbf{y}^{(J)}\right\},(\mathrm{J}=1,2, \ldots, m)$. A subset of these intersecting points is used to divide the integration domain, i.e. the local circle. It should be pointed out that the intersection points between the local and global boundaries are automatically included in $\mathbf{y}^{(J)}$. Then these special points are kept in the subset and used as the starting and ending angles. A set of angles is obtained for performing the numerical integration, as $\left\{\theta_{0}, \theta_{1}, \ldots, \theta_{t}\right\}$. The starting angle $\theta_{0}$ is not equal to the ending angle $\theta_{t}$ for the nodes on the global boundary. In the present study, the radii of the local sub-domains for the nodes within the solution domain are so chosen that the local sub-domains do not intersect with the global boundary. Hence, the subdivided angles cover the entire local circle for these internal nodes. The subdivision is illustrated in Fig. 4. In addition, the subdivision can also be used to divide the local sub-domain in pie slices with node $I$ as the center, if domain integrals are required, such as when body forces are present. Then, all the integrals over the local sub-domain can be calculated by using the simple Gaussian quadrature.

All boundary integrals are performed exactly in the same way used in the MLPG mixed finite volume method [2]. For the term of the domain integral in Eq. (11), $\int_{\Omega_{s}} \sigma_{i j} u_{i, j} d \Omega$, the integration domain is also subdivided by the same segments and two dimensional Gaussian quadrature scheme is used. The pseudo code in MatLab is implemented as,

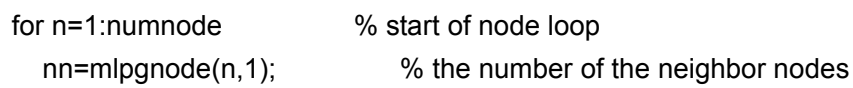

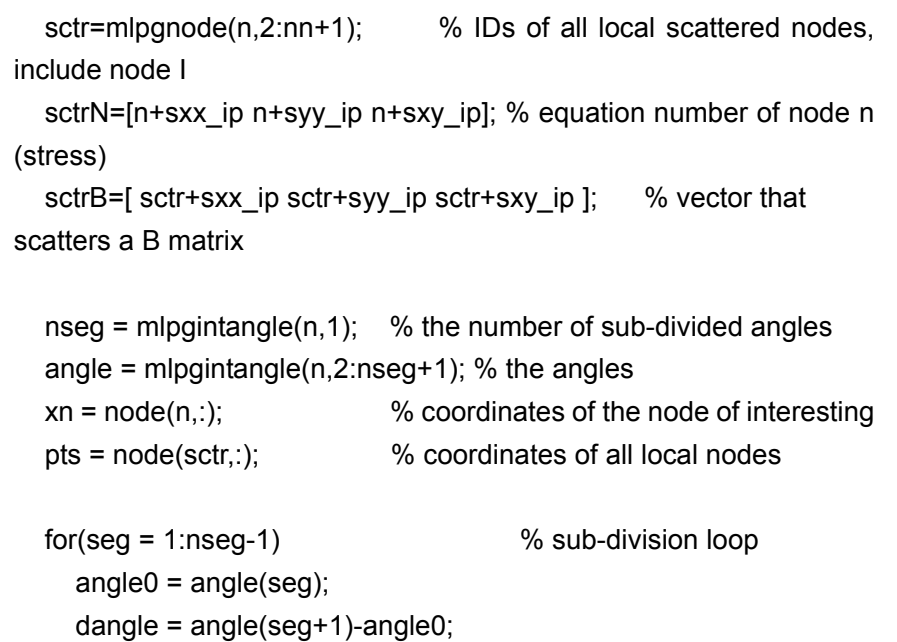

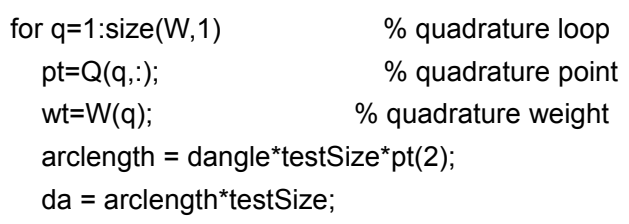

\section{NUMERICAL EXAMPLES}

2-D problems are studied in this section to illustrate the effectiveness of the present method. The numerical results of the present method, as applied to carefully chosen problems in 2D elasto-statics, specifically (i) patch test, (ii) cantilever beam, are discussed.

\section{Cube Under Uniform Tension}

The first example is that of a standard patch test, shown in Fig. 5. The material parameters are taken as $E=1.0$, and $v=$ 0.25 . The nodal configuration contains 9 nodes. Two nodal configurations are used for the testing purpose: one is regular and another is irregular, as shown in Fig. 5. In the patch tests, a uniform tensile stress is applied on the upper edge, and proper displacement constraints are applied to the lower edge. 

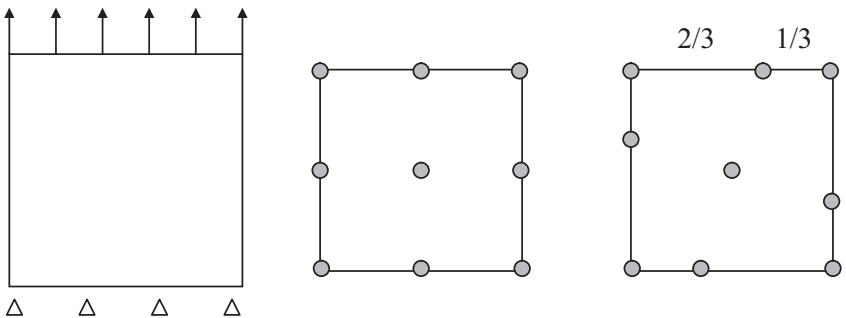

Fig. 5. A cube under uniform tension, and two nodal configurations.

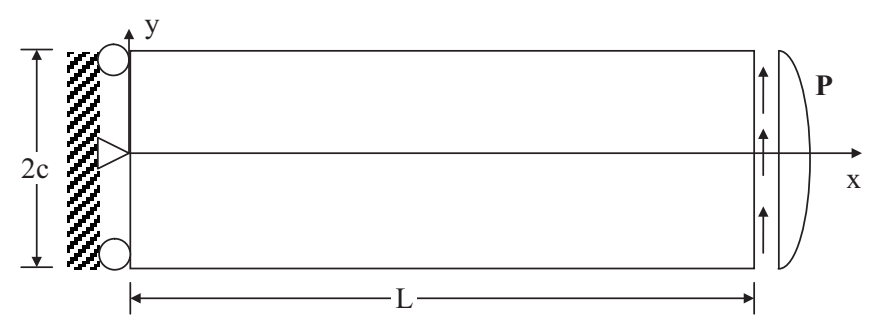

Fig. 6. A cantilever beam under an end load.

The satisfaction of the patch test requires that the displacements are linear on the lateral faces, and are constant on the upper edge; and the stresses are constant in the solution domain. It is found that the present method passes the patch tests. The maximum numerical errors are limited by the computer for two nodal configurations.

\section{Cantilever Beam}

The performances of the present MLPG formulations are also evaluated, using the problem of a cantilever beam under a transverse load, as shown in Fig. 6, for which the following exact solution is given in [23]:

$$
\begin{aligned}
& u_{x}=-\frac{P y}{6 \bar{E} I}\left[3 x(2 L-x)+(2+\bar{v})\left(y^{2}-c^{2}\right)\right] \\
& u_{y}=\frac{P}{6 \bar{E} I}\left[x^{2}(3 L-x)+3 \bar{v}(L-x) y^{2}+(4+5 \bar{v}) c^{2} x\right]
\end{aligned}
$$

where the moment of inertia $I$ the beam is given as,

$$
I=\frac{c^{3}}{3}
$$

and

$$
\bar{E}=\left\{\begin{array}{cc}
E & \bar{v}=\left\{\begin{array}{cc}
v & \text { for plane stress } \\
E /(1-v)^{2}
\end{array}\right] \text { for plane strain }
\end{array}\right.
$$

The corresponding stresses are

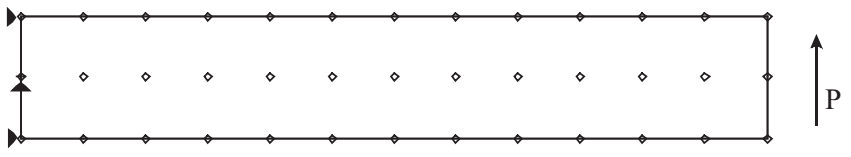

(a) $39 \operatorname{nodes}(d=2.0)$

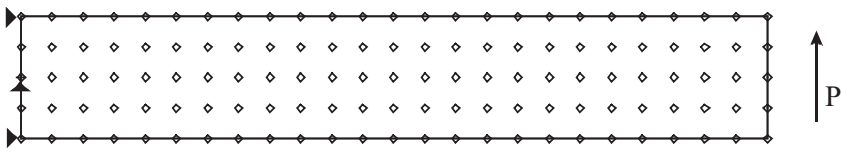

(b) 125 nodes $(d=1.0)$

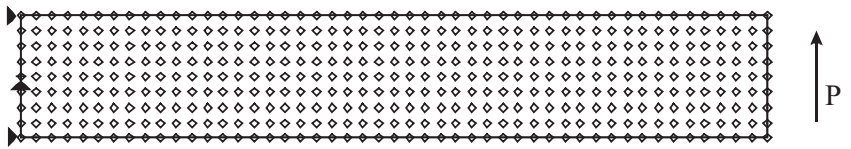

(c) 441 nodes $(d=0.5)$

Fig. 7. Three nodal configurations for a cantilever beam.

$$
\begin{aligned}
& \sigma_{x}=-\frac{P}{I}(L-x) y \\
& \sigma_{y}=0 \\
& \sigma_{x y}=-\frac{P}{2 I}\left(y^{2}-c^{2}\right)
\end{aligned}
$$

The problem is solved for the plane stress case with $P=1$, $E=1, c=2, L=24$ and $v=0.25$. Regular uniform nodal configurations with nodal distances, $d$, of $2.0,1.0$, and 0.5 are used, as shown in Fig. 7. The numbers of nodes are 39, 125, and 441 , respectively.

First, the problem is solved by using the MLS approximation, with a support size of $1.15 d$ and a local sub-domain size of $0.6 \mathrm{~d}$. The vertical displacements are shown in Fig. 8(a), (b), and (c), for the three nodal configurations, respectively. They agree with the analytical solution very well. The effects of the approximation methods, the support size, and the test-domain size are studied for the present method. The approximations are chosen to the MLS with the first order polynomials. The support size and the sub-domain size are related to the nodal distance, $d$. Normally, the ratio of the support size is greater than 1.0, to make sure that there are enough points to support the nodes on the global boundary. The ratio of the sub-domain size is chosen to be less than 1.0 in the present study.

The local sub-domain is one of the key concepts for the MLPG approach. As over-lapping sub-domains are used, the test-domain size (or the size of the sub-domain) affects the accuracy of the solution and the efficiency of the method. It is very different from the non-over-lapping methods, in which the background cells are required to partition the solution domain. In the present study, the support size is chosen to be $1.15 d$, and the test-domain size is chosen to be proportional to the nodal distance, $d$. Theoretically, the ratio is very flexible. In practice, it is chosen to be less than 1.0 to ensure that the local sub-domains of the internal nodes are entirely within the 


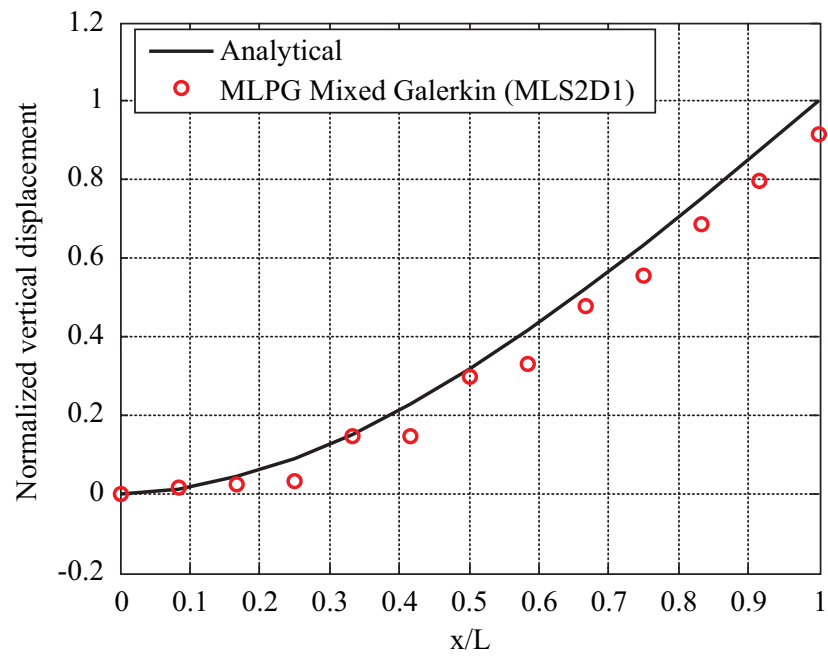

(a)

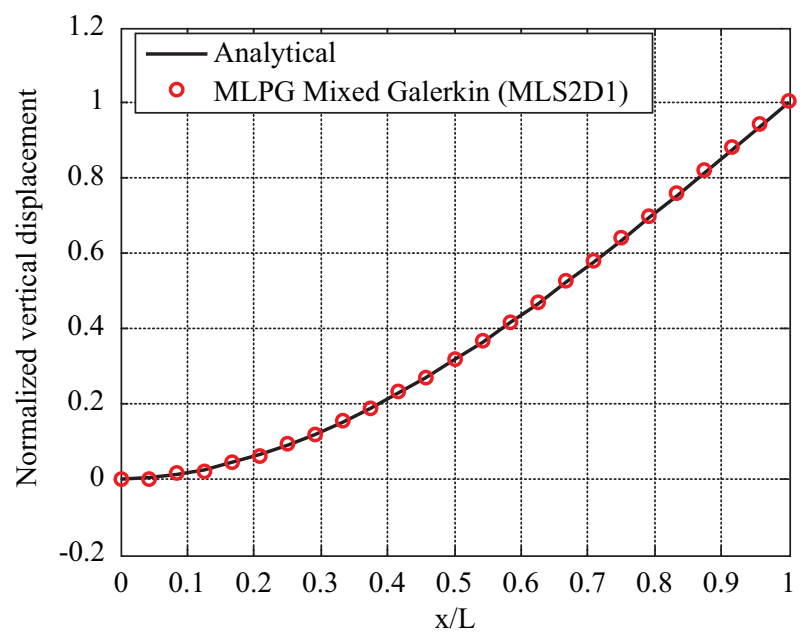

(b)

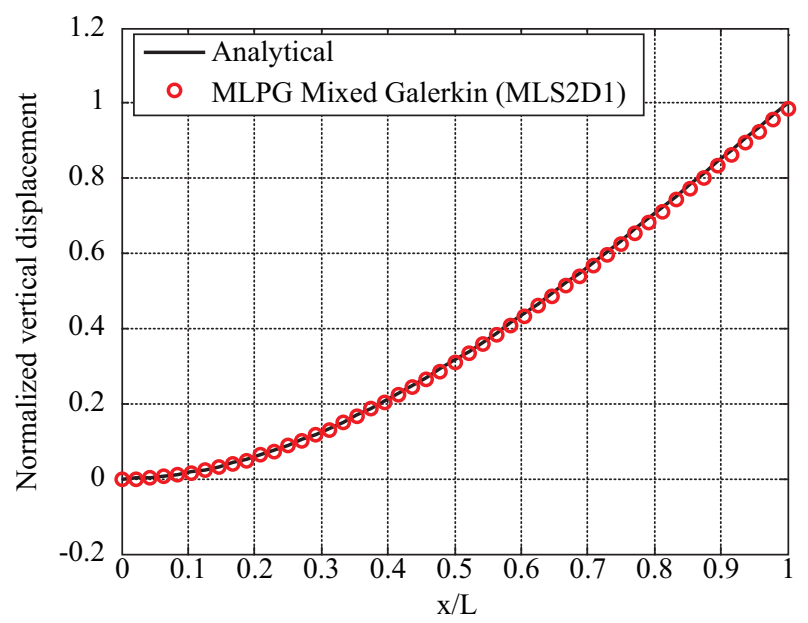

(c)

Fig. 8. (a) Normalized vertical displacement of a cantilever beam under an end loading (39 nodes with nodal distance $d=2.0$ ), (b) Normalized vertical displacement of a cantilever beam under an end loading (125 nodes with nodal distance $d=1.0$ ), (c) Normalized vertical displacement of a cantilever beam under an end loading (441 nodes with nodal distance $d=0.5$ ).

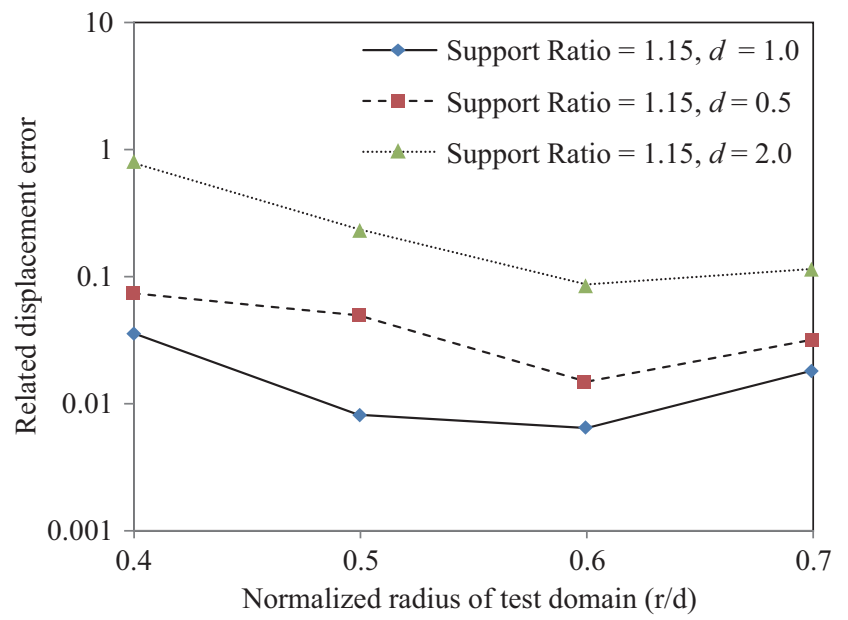

Fig. 9. Influence of the test-domain size in a cantilever beam under an end load.

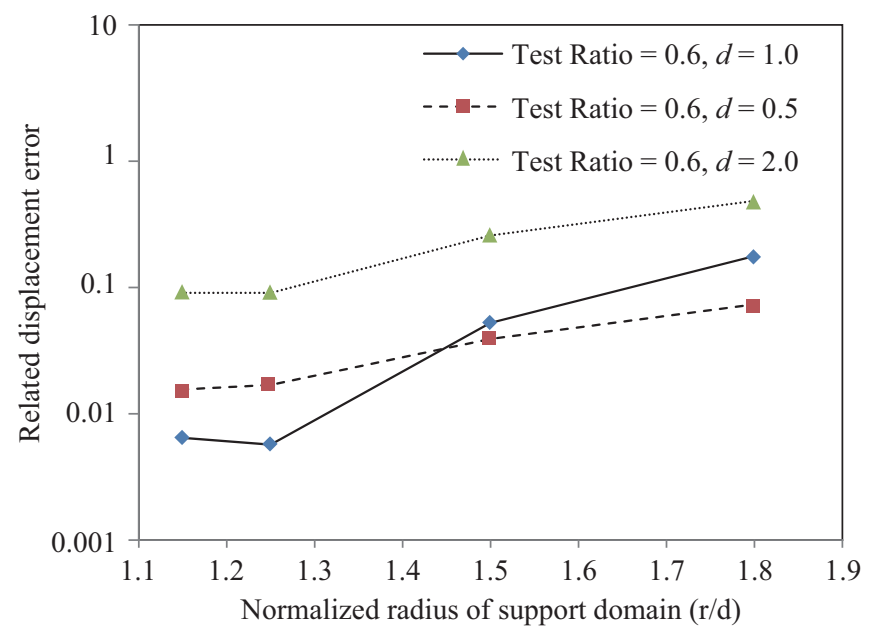

Fig. 10. Influence of the support size in a cantilever beam under an end load.

solution domain, without being intersected by the global boundary. In the present study, four ratios are used as $0.4,0.5,0.6$, and 0.7. As various test domain (local sub-domain) sizes give the reasonable results, the relative errors of the maximum displacements are used to examine the effects of the testdomain (local sub-domain where a weak-form is used) size. Three nodal configurations are used to examine the displacement errors, as shown Fig. 9. It is noticeable that the accuracy is less sensitive to the test-domain size from $0.5 \sim 0.6 d$, as the sub-domains are slightly over-lapping.

The support size (or the size of the influence domain) is also a very important parameter in meshless methods. It is related to both the accuracy of the solution, as well as the computational efficiency. For a smaller size, the meshless approximation algorithms may be singular and the shape function can not be constructed because of too few nodes. The support size is also chosen to be proportional to the nodal distance. In the 
present study, four ratios are used, as $1.15,1.25,1.5$, and 1.8 . The test size (local sub-domain) is chosen as $0.6 d$.

The relative errors of the maximum displacements are shown in Fig. 10. The numerical results show that the smaller support size gives better results, which means that the support size should be chosen as small as possible, but large enough to make sure the local MLS algorithm is not singular.

\section{CLOSURE}

A Truly Meshless Galerkin (TMG) Method has been formulated through the MLPG "Mixed" approach. The differentiation of the shape functions for displacements is totally eliminated, and the integration of the local-weak-form is performed within the local sub-domain. As a Galerkin method, the test function is chosen to be the same as the trial function. However, the local subdomain is chosen to be different in size from that of the trial -function support domain; and this makes the present MLPG Galerkin Mixed method to be quite different from, and far more general than, the classical Galerkin methods. Hence it enables the use of various combinations of the local sub-domains, which makes the present MLPG Galerkin method useful for multi-field or multi-physics analysis. The numerical results demonstrate the accuracy and stability of the present methods for solving 2-D static problems. The solutions are less sensitive to the parameters used in the MLS approximation.

\section{REFERENCES}

1. Atluri, S. N., The Meshless Local Petrov-Galerkin (MLPG) Method for Domain \& Boundary Discretizations, Tech Science Press, 680 pages (2004).

2. Atluri, S. N., Han, Z. D., and Rajendran, A. M., "A new implementation of the meshless finite volume method, through the MLPG "mixed" approach," CMES: Computer Modeling in Engineering \& Sciences, Vol. 6, No. 6, pp. 491-514 (2004).

3. Atluri, S. N., Han, Z. D., and Shen, S., "Meshless Local Patrov-Galerkin (MLPG) approaches for weakly-singular traction \& displacement boundary integral equations," CMES: Computer Modeling in Engineering \& Sciences, Vol. 4, No. 5, pp. 507-517 (2003).

4. Atluri, S. N., Kim, H. G., and Cho, J. Y., “A critical assessment of the truly Meshless Local Petrov Galerkin (MLPG) and Local Boundary Integral Equation (LBIE) methods," Computational Mechanics, Vol. 24, No. 5, pp. 348-372 (1999).

5. Atluri, S. N., Liu, H. T., and Han, Z. D., "Meshless Local Petrov-Galerkin (MLPG) mixed collocation method for elasticity problems," CMES: Computer Modeling in Engineering \& Sciences, Vol. 14, No. 3, pp. 141152 (2006).

6. Atluri, S. N., Liu, H. T., and Han, Z. D., "Meshless Local Petrov-Galerkin (MLPG) mixed finite difference method for solid mechanics," CMES: Computer Modeling in Engineering \& Sciences, Vol. 15, No. 1, pp. 1-16 (2006).
7. Atluri, S. N. and Shen, S., "The Meshless Local Petrov-Galerkin (MLPG) method: A simple \& less-costly alternative to the finite element and boundary element methods," CMES: Computer Modeling in Engineering \& Sciences, Vol. 3, No. 1, pp. 11-52 (2002).

8. Atluri, S. N. and Zhu, T., "A new Meshless Local Petrov-Galerkin (MLPG) approach in computational mechanics," Computational Mechanics, Vol. 22, pp. 117-127 (1998).

9. Han, Z. D. and Atluri, S. N., "Meshless Local Petrov-Galerkin (MLPG) approaches for solving 3D problems in elasto-statics," CMES: Computer Modeling in Engineering \& Sciences, Vol. 6, No. 2, pp. 169-188 (2004).

10. Han, Z. D. and Atluri, S. N., "A Meshless Local Petrov-Galerkin (MLPG) approach for 3-dimensional elasto-dynamics," CMC: Computers, Materials \& Continua, Vol. 1, No. 2, pp. 129-140 (2004).

11. Han, Z. D. and Atluri, S. N., "On simple formulations of weakly-singular traction \& displacement BIE, and their solutions through Petrov-Galerkin approaches," CMES: Computer Modeling in Engineering \& Sciences, Vol. 4, No. 1, pp. 5-20 (2003).

12. Han, Z. D. and Atluri, S. N., "Truly Meshless Local Petrov-Galerkin (MLPG) solutions of traction \& displacement BIEs," CMES: Computer Modeling in Engineering \& Sciences, Vol. 4, No. 6, pp. 665-678 (2003).

13. Han, Z. D. and Atluri, S. N., "A systematic approach for the development of weakly-singular BIEs," CMES: Computer Modeling in Engineering \& Sciences, Vol. 2, No. 1, pp. 41-52 (2007).

14. Jarak, T. and Soric, J., "Analysis of rectangular square plates by the mixed Meshless Local Petrov-Galerkin (MLPG) approach," CMES: Computer Modeling in Engineering \& Sciences, Vol. 38, No. 3, pp. 231-262 (2008).

15. Jarak, T., Soric, J., and Hoster, J., "Analysis of shell deformation responses by the Meshless Local Petrov-Galerkin (MLPG) approach," CMES: Computer Modeling in Engineering \& Sciences, Vol. 18, No. 3, pp. 235-246 (2007).

16. Lin, H. and Atluri, S. N., "The Meshless Local Petrov-Galerkin (MLPG) method for solving incompressible Navier-Stokes equations," CMES: Computer Modeling in Engineering \& Sciences, Vol. 2, No. 2, pp. 117 142 (2001).

17. Liu, C. S. and Atluri, S. N., "A highly accurate technique for interpolations using very high-order polynomials, and its applications to some ill-posed linear problems," CMES: Computer Modeling in Engineering \& Sciences, Vol. 43, No. 3, pp. 253-276 (2009).

18. Ma, Q. W. and Zhou, J. T. "MLPG_R method for numerical simulation of 2D breaking waves," CMES: Computer Modeling in Engineering \& Sciences, Vol. 43, No. 3, pp. 277-304 (2009).

19. Sellountos, E. J., Sequeira, A., and Polyzos, D., "Elastic transient analysis with MLPG(LBIE) method and local RBFs," CMES: Computer Modeling in Engineering \& Sciences, Vol. 41, No. 3, pp. 215-242 (2009).

20. Sladek, J., Sladek V., and Solek, P., "Elastic analysis in 3D anisotropic functionally graded solids by the MLPG," CMES: Computer Modeling in Engineering \& Sciences, Vol. 43, No. 3, pp. 223-252 (2009).

21. Sladek, J., Sladek, V., Solek, P., and Atluri, S. N., "Modeling of intelligent material systems by the MLPG," CMES: Computer Modeling in Engineering \& Sciences, Vol. 34, No. 3, pp. 273-300 (2008).

22. Sladek, J., Sladek, V., Solek, P., Tan, C. L., and Zhang, Ch., "Two- and three-dimensional transient thermoelastic analysis by the MLPG method," CMES: Computer Modeling in Engineering \& Sciences, Vol. 47, No. 1, pp. 61-96 (2009).

23. Timoshenko, S. P. and Goodier, J. N., Theory of Elasticity, 3rd edition, McGraw Hill, New York (1970).

24. Zhou, J. T. and Ma, Q. W., "MLPG method based on Rankine source solution for modelling 3D breaking waves," CMES: Computer Modeling in Engineering \& Sciences, Vol. 56, No. 2, pp. 179-210 (2010). 